\title{
Noninertial effects on the quantum dynamics of scalar bosons
}

\author{
Luis B. Castro ${ }^{\mathrm{a}}$ (1) \\ Departamento de Física, Universidade Federal do Maranhão, Campus Universitário do Bacanga, 65080-805 São Luís, MA, Brazil
}

Received: 5 November 2015 / Accepted: 21 January 2016 / Published online: 5 February 2016

(C) The Author(s) 2016. This article is published with open access at Springerlink.com

\begin{abstract}
The noninertial effect of rotating frames on the quantum dynamics of scalar bosons embedded in the background of a cosmic string is considered. In this work, scalar bosons are described by the Duffin-Kemmer-Petiau (DKP) formalism. Considering the DKP oscillator in this background the combined effects of a rotating frames and cosmic string on the equation of motion, energy spectrum, and DKP spinor are analyzed and discussed in detail. Additionally, the effect of rotating frames on the scalar bosons' localization is studied.
\end{abstract}

\section{Introduction}

The Duffin-Kemmer-Petiau (DKP) formalism [1-4] is a first-order relativistic equation that describes spin-zero and spin-one particles and has been used to analyze relativistic interactions of spin-zero and spin-one hadrons with nuclei as an alternative to their conventional second-order KleinGordon (KG) and Proca counterparts. Although the formalisms are equivalent in the case of minimally coupled vector interactions [5-7], the DKP formalism enjoys a richness of couplings that cannot be expressed in the KG and Proca theories [8,9]. Recently, there has been an increasing interest on the so-called DKP oscillator [10-19]. The DKP oscillator, on considering minimal length [20,21], noncommutative phase space [22-25], and topological defects [26] have also appeared in the literature. The DKP oscillator is a kind of tensor coupling with a linear potential which leads to the harmonic oscillator problem in the weak-coupling limit. Also, a sort of vector DKP oscillator (nonminimal vector coupling with a linear potential [27-31]) has been the topic of recent investigations. 'Vector DKP oscillator' is the name given to the system with a Lorentz vector coupling which exhibits an equally spaced energy spectrum in the weak-coupling limit.

\footnotetext{
a e-mails: bcastro.luisr@gmail.com; lrb.castro@ufma.br
}

The name distinguishes it from the system called DKP oscillator with Lorentz-tensor couplings of Refs. [10-25].

The DKP oscillator is analogous to the Dirac oscillator [32]. The Dirac oscillator is a natural model for studying properties of physical systems, it is an exactly solvable model, a variety of research efforts have been made in the context of this theoretical framework in recent years. A detailed description for the Dirac oscillator is given in Ref. [33] and for other contributions see Refs. [34-40]. The Dirac oscillator embedded in a cosmic string background has inspired a great deal of research in last years [41-47]. A cosmic string is a linear defect that changes the topology of the medium when viewed globally. The influence of this topological defect on the dynamics of spin-half particles has been widely discussed in the literature.

On the other hand, the standard description of physical phenomena according to accelerated observers is based on the hypothesis of locality, which states that an accelerated observer at each instant along its wordline is equivalent to a hypothetical inertial observer at the same event and with the same velocity as the noninertial observer. This assumption forms the basis for the extension of the Poincaré-invariant theory of relativity to general frames of references as well as gravitational fields. The study of rotating frames has discovered interesting effects, where the best-known effect is the Sagnac effect $[48,49]$. Another well-known effect is the Mashhoon effect [50], which yields a phase shift due to the coupling between the spin of the particle with the angular velocity of the rotating frames. Also we have the term due to Page-Werner et al. [51,52], which is a coupling between the angular momentum of the particle and the angular velocity of the rotating frame. Other studies of noninertial effects in quantum systems have also been extended to confined systems, for instance rotational and gravitational effects in quantum interference [53-55], scalar fields [56,57], Dirac fields [58], persistent currents in quantum rings [59], confinement of a neutral particle to a quantum dot $[60,61]$, Dirac oscillator [62,63], and spin currents [64]. Recently, the noninertial 
effects due to rotation or acceleration have been investigated in condensed matter systems as, for example, noninertial effects due to a rotating Hall sample [65], rotating BoseEinstein (BE) condensation in ultra cold diluted atomic gases [66], the effect of a rotating frame in $C_{60}$ molecules $[67,68]$, among other systems. However, investigations on noninertial effects involving scalar bosons via the DKP formalism have not been performed, therefore we believe that this problem deserves to be explored.

The main motivation of this work is to study the noninertial effects on the quantum dynamics of scalar bosons embedded in the background of a cosmic string. In this work, the influence of combined effects of the angular velocity of the rotating frame $\varpi$ and the angular deficit of the cosmic string $\alpha$ in the equation of motion, the energy spectrum, and the DKP spinor are analyzed and discussed in detail. The case of the DKP oscillator in this background is also considered. Owing to the peculiar behavior of this background, one can readily envisage that two different classes of solutions can be segregated depending on the value of the product $\varpi \alpha$. For an arbitrary value of $\varpi \alpha$ and considering the appropriate boundary condition, the possible energy levels are obtained by a root-finding procedure of a symbolic algebra program. On the other hand, for the limit $\varpi \alpha \ll 1$ and considering the appropriate boundary condition, the exact solutions are presented in closed form. We show that scalar bosons and antibosons tend to be better localized when the angular velocity of the rotating frame $\varpi$ increases. The results reported in [26] can be obtained as a particular case.

This work is organized as follows. In Sect. 2, we consider a short review on DKP equation in a curved space-time. In Sect. 3, we give a brief review on a cosmic string background, noninertial reference frame and we also analyze the curvedspace beta matrices and spin connection in this background. In Sect. 4, we concentrate our efforts on the interaction called the DKP oscillator embedded in the background of a cosmic string in a rotating coordinate system. In particular, we focus on the case of scalar bosons and obtain the equation of motion, the energy spectrum, and the DKP spinor. We analyze two kinds of solutions that depend on the value of the product $\varpi \alpha$. Finally, in Sect. 5 we present our conclusions.

\section{Review on Duffin-Kemmer-Petiau equation in a curved space-time}

The Duffin-Kemmer-Petiau (DKP) equation for a free boson in curved space-time is given by $[69,70](\hbar=c=1)$

$$
\left[i \beta^{\mu} \nabla_{\mu}-M\right] \Psi=0
$$

where the covariant derivative

$\nabla_{\mu}=\partial_{\mu}-\Gamma_{\mu}$
In this case, we restrict our analysis to the torsion-zero case and the affine connection is defined by

$\Gamma_{\mu}=\frac{1}{2} \omega_{\mu \bar{a} \bar{b}}\left[\beta^{\bar{a}}, \beta^{\bar{b}}\right]$

The curved-space beta matrices are

$\beta^{\mu}=e^{\mu}{ }_{\bar{a}} \beta^{\bar{a}}$

and satisfy the algebra

$\beta^{\mu} \beta^{v} \beta^{\lambda}+\beta^{\lambda} \beta^{v} \beta^{\mu}=g^{\mu v} \beta^{\lambda}+g^{\lambda v} \beta^{\mu}$,

where $g^{\mu \nu}$ is the metric tensor. The algebra expressed by (5) generates a set of 126 independent matrices whose irreducible representations are a trivial representation, a fivedimensional representation describing the spin-zero particles (scalar sector), and a ten-dimensional representation associated to spin-one particles (vector sector). More detailed discussions on the DKP formalism in a curved space-time can be found in Ref. [26].

The tetrads $e_{\mu}{ }^{\bar{a}}(x)$ satisfy the relations

$\eta^{\bar{a} \bar{b}}=e_{\mu}{ }^{\bar{a}} e_{\nu}{ }^{\bar{b}} g^{\mu \nu}$,

$g_{\mu \nu}=e_{\mu}{ }^{\bar{a}} e_{\nu}{ }^{\bar{b}} \eta_{\bar{a} \bar{b}}$

and

$e_{\mu}{ }^{\bar{a}} e^{\mu}{ }_{\bar{b}}=\delta_{\bar{b}}^{\bar{a}}$

the Latin indices being raised and lowered by the Minkowski metric tensor $\eta^{\bar{a} \bar{b}}$ with signature $(-,+,+,+)$ and the Greek ones by the metric tensor $g^{\mu \nu}$.

The spin connection $\omega_{\mu \bar{a} \bar{b}}$ is given by

$\omega_{\mu}^{\bar{a} \bar{b}}=e_{\alpha}{ }^{\bar{a}} e^{\nu \bar{b}} \Gamma_{\mu \nu}^{\alpha}-e^{\nu \bar{b}} \partial_{\mu} e_{\nu}^{\bar{a}}$

with $\omega_{\mu}{ }^{\bar{a}} \bar{b}=-\omega_{\mu}{ }^{\bar{b}} \bar{a}$, and $\Gamma_{\mu \nu}^{\alpha}$ are the Christoffel symbols given by

$\Gamma_{\mu \nu}^{\alpha}=\frac{g^{\alpha \beta}}{2}\left(\partial_{\mu} g_{\beta \nu}+\partial_{\nu} g_{\beta \mu}-\partial_{\beta} g_{\mu \nu}\right)$

As shown in Ref. [26], the conservation law for $J^{\mu}$ leads to

$\nabla_{\mu} J^{\mu}=\frac{1}{2} \bar{\Psi}\left(\nabla_{\mu} \beta^{\mu}\right) \Psi$

where $J^{\mu}=\frac{1}{2} \bar{\Psi} \beta^{\mu} \Psi$. The factor $1 / 2$ multiplying $\bar{\Psi} \beta^{\mu} \Psi$, of no importance regarding the conservation law, is in order to have a charge density conformable to the one used in the $\mathrm{KG}$ theory and its nonrelativistic limit [28]. The adjoint spinor $\bar{\Psi}$ 
is given by $\bar{\Psi}=\Psi^{\dagger} \eta^{0}$ with $\eta^{0}=2 \beta^{0} \beta^{0}-1$ in such a way that $\left(\eta^{0} \beta^{\mu}\right)^{\dagger}=\eta^{0} \beta^{\mu}$ (the matrices $\beta^{\mu}$ are Hermitian with respect to $\left.\eta^{0}\right)$. Thus, if

$\nabla_{\mu} \beta^{\mu}=0$,

then the four-current will be conserved. The condition (12) is the purely geometrical assertion that the curved-space beta matrices are covariantly constant.

On the other hand, the normalization condition $\int \mathrm{d} \tau J^{0}=$ \pm 1 can be expressed as

$\int \mathrm{d} \tau \bar{\Psi} \beta^{0} \Psi= \pm 2$

where the plus (minus) sign must be used for a positive (negative) charge, and the expectation value of any observable $\mathcal{O}$ can be given by

$\langle\mathcal{O}\rangle=\frac{\int \mathrm{d} \tau \bar{\Psi} \beta^{0} \mathcal{O} \Psi}{\int \mathrm{d} \tau \bar{\Psi} \beta^{0} \Psi}$,

where $\beta^{0} \mathcal{O}$ should be Hermitian with respect to $\eta^{0},\left[\eta^{0}\right.$ $\left.\left(\beta^{0} \mathcal{O}\right)\right]^{\dagger}=\eta^{0}\left(\beta^{0} \mathcal{O}\right)$, in order to provide real eigenvalues [7].

\subsection{Interaction in the Duffin-Kemmer-Petiau equation}

With the introduction of interactions, the DKP equation in a curved space-time can be written as

$$
\left(i \beta^{\mu} \nabla_{\mu}-M-U\right) \Psi=0
$$

where the more general potential matrix $U$ is written in terms of 25 (100) linearly independent matrices pertinent to a five- (ten)-dimensional irreducible representation associated to the scalar (vector) sector. The potential matrix $U$ can be written in terms of well-defined Lorentz structures. For the scalar sector (spin-zero) there are two scalar, two vector, and two tensor terms [8], whereas for the vector sector (spin-one) there are two scalar, two vector, a pseudoscalar, two pseudovector, and eight tensor terms [9].

In the presence of interaction, $J^{\mu}$ satisfies the equation

$\nabla_{\mu} J^{\mu}+\frac{i}{2} \bar{\Psi}\left(U-\eta^{0} U^{\dagger} \eta^{0}\right) \Psi=\frac{1}{2} \bar{\Psi}\left(\nabla_{\mu} \beta^{\mu}\right) \Psi$.

Thus, if $U$ is Hermitian with respect to $\eta^{0}$ and the curvedspace beta matrices are covariantly constant then the fourcurrent will be conserved. The condition (16) for the case of Minkowski space-time has been used to point out a misleading treatment in the recent literature regarding analytical solutions for nonminimal vector interactions [31].

\section{Noninertial reference frame and the cosmic string background}

The cosmic string space-time is an object described by the line element

$\mathrm{d} s^{2}=-\mathrm{d} T^{2}+\mathrm{d} R^{2}+\alpha^{2} R^{2} \mathrm{~d} \Phi^{2}+\mathrm{d} Z^{2}$

where $-\infty<Z<+\infty, R \geq 0$, and $0 \leq \Phi \leq 2 \pi$. The parameter $\alpha$ is associated with the linear mass density $\tilde{m}$ of the string by $\alpha=1-4 \tilde{m}$ and runs in the interval $(0,1]$ and corresponds to a deficit angle $\gamma=2 \pi(1-\alpha)$. In the geometric context, the line element (19) is related to a Minkowski space-time with a conical singularity [71]. Note that in the limit as $\alpha \rightarrow 1$ we obtain the line element of cylindrical coordinates.

The rotating frame is obtained using the following coordinate transformation:

$T=t, \quad R=r, \quad \Phi=\varphi+\varpi t, \quad Z=z$,

where $\varpi$ is the constant angular velocity of the rotating frame. So, the line element (17) becomes

$$
\begin{aligned}
\mathrm{d} s^{2}= & -\left(1-\varpi^{2} \alpha^{2} r^{2}\right) \mathrm{d} t^{2}+2 \varpi \alpha^{2} r^{2} \mathrm{~d} \varphi \mathrm{d} t \\
& +\mathrm{d} r^{2}+\alpha^{2} r^{2} \mathrm{~d} \varphi^{2}+\mathrm{d} z^{2}
\end{aligned}
$$

This line element describes the background of a cosmic string in a rotating coordinate system. It is worthwhile to mention that the line element (19) is defined in the interval $0<r<r_{0}$, where $r_{0}=1 / \varpi \alpha$ and that values of $r>r_{0}$ correspond to a particle placed outside of the line cone. This interesting fact imposes one restriction on the radial coordinate: the wave function of the quantum particle must vanish at $r \rightarrow r_{0}$. This peculiar behavior can be interpreted in such a way that the geometry of the space-time plays the role of a hard-wall confining potential $[60,61,72-74]$.

The basis tetrad $e^{\mu}{ }_{\bar{a}}$ from the line element (19) is chosen to be

$e^{\mu}{ }_{\bar{a}}=\left(\begin{array}{cccc}\frac{1}{\sqrt{1-\rho^{2}}} & 0 & \frac{\varpi \alpha r}{\sqrt{1-\rho^{2}}} & 0 \\ 0 & 1 & 0 & 0 \\ 0 & 0 & \frac{\sqrt{1-\rho^{2}}}{\alpha r} & 0 \\ 0 & 0 & 0 & 1\end{array}\right)$,

where $\rho=\varpi \alpha r$. For the specific basis tetrad (20) the curvedspace beta matrices read 
$\beta^{0}=\frac{1}{\sqrt{1-\rho^{2}}}\left(\beta^{\overline{0}}+\varpi \alpha r \beta^{\overline{2}}\right)$,

$\beta^{r}=\beta^{\overline{1}}$,

$\beta^{\varphi}=\frac{\sqrt{1-\rho^{2}}}{\alpha r} \beta^{\overline{2}}$,

$\beta^{z}=\beta^{\overline{3}}$,

and thereby, for the covariant derivative one gets

$\nabla_{0}=\partial_{0}-\Gamma_{0}$,

$\nabla_{r}=\partial_{r}-\Gamma_{r}$,

$\nabla_{\varphi}=\partial_{\varphi}-\Gamma_{\varphi}$,

$\nabla_{z}=\partial_{z}$,

where the spin connections are given by

$$
\begin{aligned}
& \Gamma_{0}=\frac{\varpi \alpha}{\sqrt{1-\rho^{2}}}\left(\varpi \alpha r\left[\beta^{\overline{0}}, \beta^{\overline{1}}\right]-\left[\beta^{\overline{1}}, \beta^{\overline{2}}\right]\right), \\
& \Gamma_{r}=-\frac{\varpi \alpha}{1-\rho^{2}}\left[\beta^{\overline{0}}, \beta^{\overline{2}}\right], \\
& \Gamma_{\varphi}=\frac{\Gamma_{0}}{\varpi} .
\end{aligned}
$$

Note that using the line element (19) and the representation for the curved-space beta matrices (21)-(24) the condition (12) is satisfied and therefore the current is conserved for this background.

\section{DKP oscillator in a noninertial reference frame}

In this section, we concentrate our efforts on the interaction called a DKP oscillator. For this external interaction we use the nonminimal substitution [11]

$\mathbf{p} \rightarrow \mathbf{p}-i M \omega \eta^{0} \mathbf{r}$

where $\omega$ is the oscillator frequency. This interaction is of Lorentz-tensor type and is Hermitian with respect to $\eta^{0}$, so it furnishes a conserved four-current. Considering only the radial component for the nonminimal substitution one gets

$\mathbf{p} \rightarrow \mathbf{p}-i M \omega \eta^{0} r \hat{r}$

As the interaction is time-independent one can write $\Psi(\mathbf{r}, t)$ $=\Phi(\mathbf{r}) \exp (-i E t)$, where $E$ is the energy of the scalar boson, in such a way that the time-independent DKP equation becomes

$$
\begin{gathered}
{\left[\beta^{0}\left(E-\Gamma_{0}\right)+i \beta^{\overline{1}}\left(\partial_{r}-\Gamma_{r}+M \omega \eta^{0} r\right)\right.} \\
\left.+i \beta^{\varphi}\left(\partial_{\varphi}-\Gamma_{\varphi}\right)+i \beta^{\overline{3}} \partial_{z}-M\right] \Phi=0
\end{gathered}
$$

where $\beta^{0}, \beta^{\varphi}, \Gamma_{0}, \Gamma_{r}$, and $\Gamma_{\varphi}$ are given by (21), (23), (29), (30), and (31), respectively.

\subsection{Scalar sector}

For the case of scalar bosons (scalar sector), we use the standard representation for the beta matrices given by [75]

$\beta^{\overline{0}}=\left(\begin{array}{cc}\theta & \overline{0} \\ \overline{0}^{T} & \mathbf{0}\end{array}\right), \quad \vec{\beta}=\left(\begin{array}{cc}\widetilde{0} & \vec{\sigma} \\ -\vec{\sigma}^{T} & \mathbf{0}\end{array}\right)$

where

$$
\begin{gathered}
\theta=\left(\begin{array}{ll}
0 & 1 \\
1 & 0
\end{array}\right), \quad \sigma^{1}=\left(\begin{array}{ccc}
-1 & 0 & 0 \\
0 & 0 & 0
\end{array}\right) \\
\sigma^{2}=\left(\begin{array}{lll}
0 & -1 & 0 \\
0 & 0 & 0
\end{array}\right), \quad \sigma^{3}=\left(\begin{array}{ccc}
0 & 0 & -1 \\
0 & 0 & 0
\end{array}\right)
\end{gathered}
$$

$\overline{0}, \tilde{0}$, and $\mathbf{0}$ are $2 \times 3,2 \times 2$, and $3 \times 3$ zero matrices, respectively, while the superscript $\mathrm{T}$ designates matrix transposition. The five-component spinor can be written as $\Phi^{T}=\left(\Phi_{1}, \ldots, \Phi_{5}\right)$ and the DKP equation for scalar bosons becomes

$$
\begin{gathered}
\frac{E}{\sqrt{1-\rho^{2}}} \Phi_{2}-M \Phi_{1}-i\left(\partial_{-}+\frac{1}{r}\right) \Phi_{3}-i \partial_{z} \Phi_{5} \\
-\frac{\varpi \alpha r}{M \sqrt{1-\rho^{2}}}\left(E+\frac{i\left(1-\rho^{2}\right)}{\varpi \alpha^{2} r^{2}} \partial_{\varphi}\right) \Phi_{4}=0,
\end{gathered}
$$

$\Phi_{2}=\frac{E}{M \sqrt{1-\rho^{2}}} \Phi_{1}$,

$\Phi_{3}=\frac{i}{M} \partial_{+} \Phi_{1}$

$\Phi_{4}=\frac{\varpi \alpha r}{M \sqrt{1-\rho^{2}}}\left(E+\frac{i\left(1-\rho^{2}\right)}{\varpi \alpha^{2} r^{2}} \partial_{\varphi}\right) \Phi_{1}$,

$\Phi_{5}=\frac{i}{M} \partial_{z} \Phi_{1}$

where

$\partial_{-}=\partial_{r}-M \omega r$,

$\partial_{+}=\partial_{r}+M \omega r$,

Meanwhile,

$$
\begin{aligned}
J^{0} & =\frac{1}{\sqrt{1-\rho^{2}}}\left[\operatorname{Re}\left(\Phi_{2}^{*} \Phi_{1}\right)-\rho \operatorname{Re}\left(\Phi_{4}^{*} \Phi_{1}\right)\right] \\
& =\frac{E\left|\Phi_{1}\right|^{2}+\varpi \operatorname{Re}\left(i \Phi_{1} \partial_{\varphi} \Phi_{1}^{*}\right)}{M} .
\end{aligned}
$$


Combining these results we obtain the equation of motion for the first component of the DKP spinor,

$$
\begin{aligned}
& {\left[\nabla_{\alpha}^{2}-M^{2} \omega^{2} r^{2}-2 i E \varpi \partial_{\varphi}-\varpi^{2} \partial_{\varphi}^{2}\right.} \\
& \left.+E^{2}-M^{2}+2 M \omega\right] \Phi_{1}=0
\end{aligned}
$$

where $\nabla_{\alpha}^{2}$ is the Laplace-Beltrami operator in the conical space, given by

$\nabla_{\alpha}^{2}=\frac{1}{r} \frac{\partial}{\partial r}\left(r \frac{\partial}{\partial r}\right)+\frac{1}{\alpha^{2} r^{2}} \frac{\partial^{2}}{\partial \varphi^{2}}+\frac{\partial^{2}}{\partial z^{2}}$.

At this stage, we can use the invariance under boosts along the $z$-direction and adopt the usual decomposition

$\Phi_{1}(r, \varphi, z)=\frac{\phi_{1}(r)}{\sqrt{r}} e^{i l \varphi+i k_{z} z}$

with $l=0, \pm 1, \pm 2, \ldots$ Inserting this into Eq. (45), we get

$$
\left[\frac{\mathrm{d}^{2}}{\mathrm{~d} r^{2}}-\lambda^{2} r^{2}-\frac{\left(l_{\alpha}^{2}-\frac{1}{4}\right)}{r^{2}}+\kappa^{2}\right] \phi_{1}=0
$$

where $l_{\alpha}=l / \alpha, \lambda=M \omega$, and

$\kappa=\sqrt{(E+\varpi l)^{2}-M^{2}+2 M \omega-k_{z}^{2}}$.

The equation of motion (48) describes the quantum dynamics of a DKP oscillator in the backgroud of a cosmic string in a rotating coordinate system. The solution close to the origin valid for all values of $l_{\alpha}$ can be written as being proportional to $r^{\left|l_{\alpha}\right|+\frac{1}{2}}$. On the other hand, for sufficiently large radius $r_{0}$ the square-integrable solution behaves as $e^{-\lambda r^{2} / 2}$, thereby the solution for $0<r<r_{0}$ can be expressed as

$\phi_{1}(r)=r^{\left|l_{\alpha}\right|+\frac{1}{2}} e^{-\lambda r^{2} / 2} f(r)$,

and subsequently, by introducing the following new variable and parameters:

$\xi=\lambda r^{2}$,

$a=\frac{1}{2}\left(\left|l_{\alpha}\right|+1-\frac{\kappa^{2}}{2 \lambda}\right)$,

$b=\left|l_{\alpha}\right|+1$,

one finds that $f(\xi)$ can be expressed as a regular solution of the confluent hypergeometric equation (Kummer's function) [76],

$\xi \frac{\mathrm{d}^{2} f}{\mathrm{~d} \xi^{2}}+(b-\xi) \frac{\mathrm{d} f}{\mathrm{~d} \xi}-a f=0$.
The general solution of (54) is given by [76]

$f(\xi)=A M(a, b, \xi)+B \xi^{1-b} M(a-b+1,2-b, \xi)$

where $A$ and $B$ are arbitrary constants. The second term in (55) has a singular point at $\xi=0$, so that we set $B=0$. Thus the solution for (54) is given by

$f(\xi)=A M(a, b, \xi)$

As mentioned in Sect. 3, for the peculiar behavior of this background, which is defined in the interval $0<r<r_{0}$, where $r_{0}=1 / \varpi \alpha$, the problem presents two different classes of solutions that depend on the value of the product $\varpi \alpha$. Let us consider as a first case an arbitrary value of $\varpi \alpha$ and as a second case the limit $\varpi \alpha \ll 1$. In the two next sections, we will analyze each case in detail.

\subsection{Arbitrary $\varpi \alpha$}

Following the discussions of Sect. 3, we proceed now to find the eigenfunction for this problem. Because of the restriction on the radial coordinate due to noninertial effects a physical solution is possible only if the eigenfunction vanishes at $r=$ $r_{0}=1 / \varpi \alpha$ in order to normalize $\phi_{1}$; thereby the boundary condition implies that

$M\left(a, b, \frac{\lambda}{\varpi^{2} \alpha^{2}}\right)=0$.

By solving this quantization condition one obtains the possible energy levels by inserting the allowed values of $a=a_{l}$ in (52), and combining with (49) yields

$E_{ \pm}= \pm \sqrt{2 M \omega\left(\frac{|l|}{\alpha}-2 a_{l}\right)+M^{2}+k_{z}^{2}}-\varpi|l|$,

which is irrespective of the sign of the angular momentum quantum number $|l|$. From (58) we can see that the discrete set of DKP energies is composed of two contributions: the first term of (58) is associated to the DKP oscillator embedded in a cosmic string background and the second term of (58) is associated to the noninertial effect of rotating frames, which in turn is a Sagnac-type effect $[48,49]$. Note that both particle $\left(E_{+}\right)$and antiparticle $\left(E_{-}\right)$energy levels are members of the spectrum and also that the noninertial effect is to break the symmetry of the energy spectrum about $E=0$. From (58) we can conclude that $\left|E_{-}\right|>\left|E_{+}\right|$. Furthermore, if $|l|=0$ or $\varpi=0$ the discrete set of DKP energies are symmetrical about $E=0$. At this stage, we can use the invariance under boosts along the $z$-direction, and without loss of generality we can fix $k_{z}=0$. 
Table 1 The first values of $a_{l}$ that satisfy the quantization condition (57) for $\omega=0.1$ and $\varpi=0.5$
Table 2 The first values of $a_{l}$ that satisfy the quantization condition (57) for $\omega=0.1$, and $\varpi=1.0$

\begin{tabular}{|c|c|c|c|c|c|}
\hline \multirow[b]{3}{*}{$\alpha$} & \multicolumn{5}{|c|}{$M\left(a=a_{l}, b, \frac{\lambda}{\varpi^{2} \alpha^{2}}\right)=0$} \\
\hline & \multicolumn{2}{|l|}{$l=0$} & \multicolumn{3}{|l|}{$l=1$} \\
\hline & $a_{l}$ & $\left|E_{ \pm}\right|$ & $a_{l}$ & $E_{+}$ & $E_{-}$ \\
\hline \multirow[t]{5}{*}{0.9} & -2.4546 & 1.4078 & -7.0092 & 1.5065 & -2.5065 \\
\hline & -14.9645 & 2.6431 & -25.0484 & 2.8528 & -3.8528 \\
\hline & -37.4516 & 3.9976 & -53.0832 & 4.2387 & -5.2387 \\
\hline & -69.9297 & 5.3826 & -91.1117 & 5.6373 & -6.6373 \\
\hline & -112.4003 & 6.7794 & -139.1333 & 7.0416 & -8.0416 \\
\hline \multirow[t]{5}{*}{0.5} & -0.4896 & 1.0935 & -2.7840 & 1.0854 & -2.0854 \\
\hline & -4.3861 & 1.6596 & -9.7150 & 1.7991 & -2.7991 \\
\hline & -11.3311 & 2.3521 & -19.7364 & 2.5487 & -3.5487 \\
\hline & -21.3566 & 3.0891 & -32.8433 & 3.3128 & -4.3128 \\
\hline & -34.4654 & 3.8453 & -49.0351 & 4.0841 & -5.0841 \\
\hline \multirow[t]{5}{*}{0.1} & 0.0000 & 1.0000 & 0.0000 & 1.2321 & -2.2321 \\
\hline & -1.0000 & 1.1832 & -1.0000 & 1.3439 & -2.3439 \\
\hline & -2.0000 & 1.3416 & -2.0006 & 1.4494 & -2.4494 \\
\hline & -3.0000 & 1.4832 & -3.0075 & 1.5501 & -2.5501 \\
\hline & -4.0000 & 1.6125 & -4.0476 & 1.6492 & -2.6492 \\
\hline
\end{tabular}

Although the quantization condition has no closed form solutions in terms of simpler functions, the numerical computation of $a_{l}$ can be done easily with a root-finding procedure of a symbolic algebra program. The first values of $a_{l}$ that satisfy the quantization condition (57) and its respective energies are listed in Tables 1 and 2 for $\varpi=0.5$ and $\varpi=1.0$, respectively.
With all that, the solution for $0<r<r_{0}$ can be written as $\phi_{1}(r)=A_{l} r^{\left|l_{\alpha}\right|+\frac{1}{2}} e^{-\lambda r^{2} / 2} M\left(a_{l}, b, \lambda r^{2}\right)$,

where $A_{l}$ is a normalization constant. The charge density $J^{0}$ (44) dictates that $\phi_{1}$ must be normalized as

$$
\frac{\left|E_{ \pm}+\varpi\right| l||}{M} \int_{0}^{r_{0}} \mathrm{~d} r\left|\phi_{1}\right|^{2}=1
$$




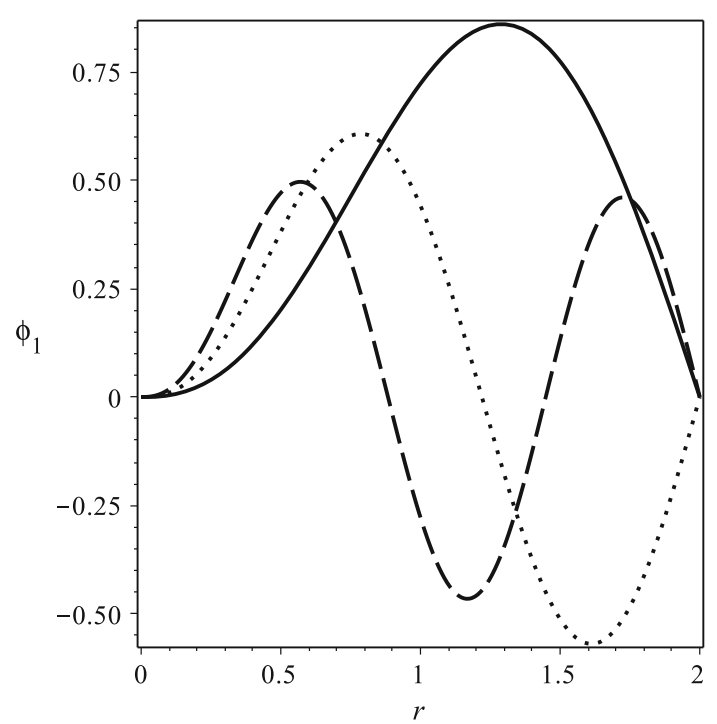

Fig. 1 Plots of $\phi_{1}$ (normalized) for the ground state (solid line), first excited state (dotted line), and second excited state (dashed line) for $\omega=0.1,|l|=1, \alpha=0.5$, and $\varpi=1.0$

so that the normalization constant can be written as

$A_{l}=\sqrt{\frac{M}{\delta\left|E_{ \pm}+\varpi\right| l||}}$,

where

$\delta=\int_{0}^{r_{0}} \mathrm{~d} r r^{2\left|l_{\alpha}\right|+1} e^{-\lambda r^{2}}\left|M\left(a_{l}, b, \lambda r^{2}\right)\right|^{2}$

with $\left|E_{ \pm}+\varpi\right| l|| \neq 0$. From (61) we can see that $\mid E_{+}+$ $\varpi|l||=| E_{-}+\varpi|l| \mid$ as expected and that $A_{l}$ for particles is equal to $A_{l}$ for antiparticles. Figure 1 illustrates the behavior of $\phi_{1}$ (normalized) for the three lowest states, $\omega=0.1$, $|l|=1, \alpha=0.5$, and $\varpi=1.0$. Here, we consider only bosons, i.e. $E_{+}$. Note that $\varpi \alpha=0.5$, so we see that the solution is restricted to the interval $0<r<2$. Also, we can note that the solution for the ground state has no nodes, the first excited state has one node, and the second excited state has two nodes. From this fact we can conclude that there exists a systematic relation between the number of nodes of $\phi_{1}$ and each level of states (usual node structure), even if there is a restriction at the radial variable. In another context, a similar restriction in a one-dimensional problem was studied in [77].

In Figs. 2 and 3, we illustrate the results of $\left|\phi_{1}\right|^{2}$ for the ground state and the second excited state, $\omega=0.1, \alpha=$ 0.5 , and two different values of $\varpi$. Figures 2 and 3 clearly show the noninertial effects on the excited states, which are qualitatively similar to $|l|=0$ and $|l|=1$, respectively. From Fig. 2 one can see that for the ground state, the distribution has a maximum at $r \approx 1.5$ for $\varpi=0.5$ and $|l|=0$, and this

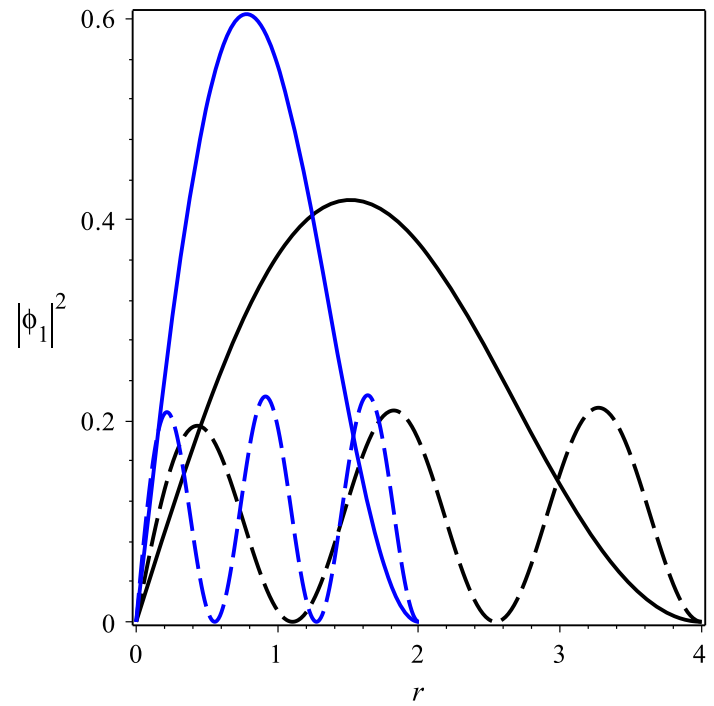

Fig. 2 Plots of $\left|\phi_{1}\right|^{2}$ for the ground state (solid line) and the second excited state (dashed line), $\omega=0.1,|l|=0, \alpha=0.5$, and for $\varpi=0.5$ (black) and $\varpi=1.0$ (blue)

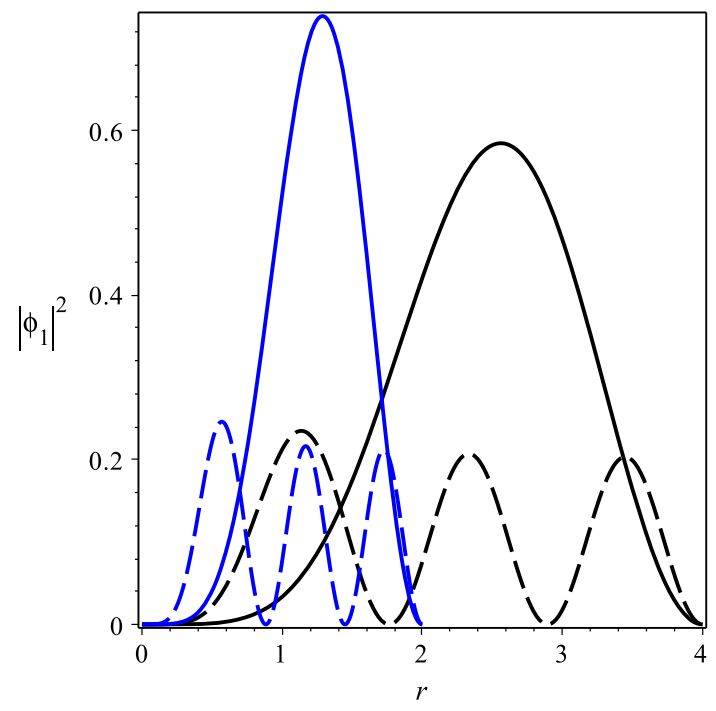

Fig. 3 The same as Fig. 2, for $|l|=1$.

maximum increases and moves to the negative $r$-direction as $\varpi=1.0$.

Then, for the study the scalar bosons' localization we use (14), and the quantity $(\Delta x)^{2}=\left\langle x^{2}\right\rangle-\langle x\rangle^{2}$ can be written as [28]

$(\Delta x)^{2}=\int_{0}^{r_{0}} \mathrm{~d} r\left|J^{0}\right|^{2} r^{2}-\left(\int_{0}^{r_{0}} \mathrm{~d} r\left|J^{0}\right| r\right)^{2}$.

Calculating $\Delta x$ for the parameter set of Fig. $2(\omega=0.1$, $|l|=0$, and $\alpha=0.5$ ), we obtain $\Delta x=0.7773(\varpi=$ $0.5)$ and $\Delta x=0.3909(\varpi=1.0)$ for the ground state and $\Delta x=1.1477(\varpi=0.5)$ and $\Delta x=0.5740(\varpi=1.0)$ for the second excited state. A similar behavior is observed at 
$\Delta x$ for the parameter set of Fig. $3(\omega=0.1,|l|=1$, and $\alpha=0.5)$. In this case, we obtain $\Delta x=0.5904(\varpi=0.5)$ and $\Delta x=0.2946(\varpi=1.0)$ for the ground state and $\Delta x=$ $1.0019(\varpi=0.5)$ and $\Delta x=0.5005(\varpi=1.0)$ for the second excited state. From these results, we can conclude that scalar bosons tend to be better localized when $\varpi$ increases. If instead of considering bosons, we consider antibosons (i.e. $E_{-}$), one would expect the same results, since $A_{l}$ for particles is equal to $A_{l}$ for antiparticles.

\subsection{Limit $\varpi \alpha \ll 1\left(r_{0} \rightarrow \infty\right)$}

The second class of solutions is obtained adopting the limit $\varpi \alpha \ll 1$. The main consequence is a change to the boundary condition and the restriction on the radial coordinate due to noninertial effects. Considering a large $r_{0}\left(r_{0} \rightarrow \infty\right)$ the square-integrable solution is guaranteed by the term $e^{-\lambda r^{2} / 2}$ in (50) and without loss generality the boundary condition becomes

$M\left(a, b, \frac{\lambda}{\varpi^{2} \alpha^{2}} \rightarrow \infty\right)=$ finite.

Now we need to analyze the asymptotic behavior of the solution (56).

The asymptotic behavior of Kummer's function is dictated by

$M(a, b, \xi) \simeq \frac{\Gamma(b)}{\Gamma(b-a)} e^{-i \pi a} \xi^{-a}+\frac{\Gamma(b)}{\Gamma(a)} e^{\xi} \xi^{a-b}$.

It is true that the presence of $e^{\xi}$ in the second term of (65) perverts the normalizability of $\phi_{1}(\xi)$ in (50). Nevertheless, this unfavorable behavior can be remedied by demanding $a=-n$, where $n$ is a nonnegative integer and $b \neq-\tilde{n}$, where $\tilde{n}$ is also a nonnegative integer. As a matter of fact, $M(-n, b, \xi)$ with $b>0$ is proportional to the generalized Laguerre polynomial $L_{n}^{(b-1)}(\xi)$, a polynomial of degree $n$ with $n$ distinct positive zeros in the range $[0, \infty)$. Therefore, the solution for all $r$ can be written as

$\phi_{1}(r)=A_{n} r^{\left|l_{\alpha}\right|+\frac{1}{2}} e^{-\lambda r^{2} / 2} L_{n}^{\left|l_{\alpha}\right|}\left(\lambda r^{2}\right)$,

where $A_{n}$ is a normalization constant. Again, the charge density $J^{0}(44)$ dictates that $\phi_{1}$ must be normalized as

$\frac{\left|E_{ \pm}+\varpi\right| l||}{M} \int_{0}^{\infty} \mathrm{d} r\left|\phi_{1}\right|^{2}=1$

so that the normalization constant can be written as

$A_{n}=\sqrt{\frac{2 M \lambda^{\left|l_{\alpha}\right|+1} \Gamma(n+1)}{\left|E_{ \pm}+\varpi\right| l|| \Gamma\left(\left|l_{\alpha}\right|+n+1\right)}}$ with $\left|E_{ \pm}+\varpi\right| l|| \neq 0$. Moreover, the requirement $a=-n$ (quantization condition) implies

$E_{ \pm}= \pm \sqrt{2 M \omega\left(2 n+\frac{|l|}{\alpha}\right)+M^{2}+k_{z}^{2}}-\varpi|l|$.

Similar to the case of arbitrary $\varpi \alpha$, the discrete set of DKP energies is modified by the term $\varpi|l|$. This last result shows that the discrete set of DKP energies are not symmetrical about $E=0$. Here the same discussion is applicable as for the energy spectrum (58). As a particular case, setting $\varpi=0$ we obtain the DKP energies of the DKP oscillator embedded in the background of a cosmic string, already reported in [26]. Again, due to invariance under rotation along the $z$-direction, without loss of generality we can fix $k_{z}=0$.

Now, let us consider the nonrelativistic limit of (69). Following the standard procedure, $E=M+\mathcal{E}$ with $M \gg \mathcal{E}$, and after some calculations one has

$\mathcal{E} \simeq \omega\left(2 n+\frac{|l|}{\alpha}\right)-\varpi|l|$

This last result describes the energy of a traditional nonrelativistic harmonic oscillator plus the Page-Werner term [51,52].

Figure 4 illustrates the profile of the energy as a function of $\omega$ for $|l|=2$. In this figure we consider the four first quantum numbers. From Fig. 4 one sees that all the energy levels emerge from the positive (negative)-energy continuum so that it is plausible to identify them with particle (antiparticle) levels.

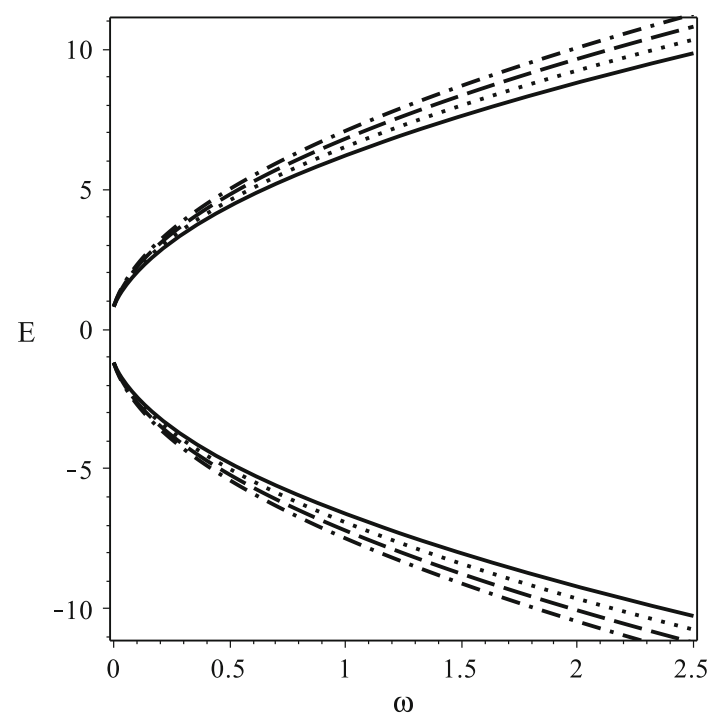

Fig. 4 Plots of the energy as a function of $\omega$ for $|l|=2$, $\varpi=0.1$, $\alpha=0.1$, and different values of $n$. For $n=0$ (solid line), $n=1$ (dotted line), $n=2$ (dashed line), and $n=3$ (dot-dashed line) 
Finally, our results for the limit $\varpi \alpha \ll 1$ are consistent with our results for arbitrary $\varpi \alpha$; this fact can be seen from Table 1 . In this table, the product $\varpi \alpha=0.05$ can be considered to be $\ll 1$, because $a_{l} \rightarrow-n$ for $|l|=0$ or $|l|=1$, where $n$ is a nonnegative integer. This means that the choice of $\varpi=0.5$ and $\alpha=0.1$, which produces $r_{0}=20$ is suitable, because this $r_{0}$ can be considered as a sufficiently large radius.

\section{Conclusions}

We studied the Duffin-Kemmer-Petiau (DKP) equation in a cosmic string background in a rotating reference frame. We showed that considering this background and a DKP oscillator interaction, they furnish a conserved current. Considering only scalar bosons, we calculated the equation of motion, which describes the quantum dynamics of a DKP oscillator in this background, and we discussed in detail the combined effects of the angular velocity of the rotating frame $\varpi$ and the angular deficit of the cosmic string background $\alpha$. This problem was mapped into a confluent hypergeometric equation for the first component of the DKP spinor $\phi_{1}$ and the remaining components were expressed in terms of the first one in a simple way.

Due to the peculiar behavior of this background, which is defined in the interval $0<r<r_{0}$, where $r_{0}=1 / \varpi \alpha$, the problem presents two kinds of solutions, depending on the value of the product $\varpi \alpha$. As a first case, we considered an arbitrary value of $\varpi \alpha$. Using the appropriate boundary condition at $r=r_{0}$, we obtained the possible energy levels by a root-finding procedure of a symbolic algebra program. The first component of the DKP spinor $\phi_{1}$ was expressed in terms of the Kummer function. As a second case, we considered the limit $\varpi \alpha \ll 1$, which implies $r_{0} \rightarrow \infty$ (a sufficiently large radius). Again, using the appropriate boundary condition at $r=r_{0} \rightarrow \infty$, we obtained the energy levels in analytic form. In this case, $\phi_{1}$ was expressed in terms of the generalized Laguerre polynomial. In both kinds of solutions, we showed that there exists a systematic relation between the number of nodes of $\phi_{1}$ and each level of states (usual node structure), even if there is a restriction on the radial variable.

For both kinds of solutions, we found the energy spectrum for this problem. We showed that the discrete set of DKP energies is composed of two contributions. One term is associated to the DKP oscillator embedded in a cosmic string background and the other term is associated to the noninertial effect of rotating reference frames, which in turn is a Sagnactype effect. Both particle $\left(E_{+}\right)$and antiparticle $\left(E_{-}\right)$energy levels are members of the spectrum, and, also, the noninertial effect breaks the symmetry of the energy spectrum about $E=0$. Only for $|l|=0$ the energy spectrum is symmetrical about $E=0$. In general, we showed that $\left|E_{-}\right|>\left|E_{+}\right|$ and that bosons as well as antibosons tend to be better localized as $\varpi$ increases. We obtained the results reported in [26] as a particular case, setting $\varpi \rightarrow 0$. We also found that the nonrelativistic limit furnishes the energy of a traditional nonrelativistic harmonic oscillator plus the Page-Wenner term. Further, we showed that both kinds of solutions are consistent when a suitable $r_{0}$ (sufficiently large) is chosen.

Beyond investigating the quantum dynamics of scalar bosons, the results of this paper could be used, in principle, in condensed matter physics. The analogy is well known between cosmic strings and disclinations in solids [78]; this fact is associated to the metric which describes a disclination corresponding to the spatial part of the line element of the cosmic string. Thereby, our results could be used to investigate the integer quantum Hall effect for bosons [79] in a system in the presence of a topological defect in a rotating frame as done in [65] for the quantum Hall effect, and they could also be used to investigate the symmetry-protected topological (SPT) phase [80], which is the analog of the celebrated free fermion topological insulator for a bosonic system. In this context, the DKP theory has been employed on the study of novel topological semimetals [81]. Another physical application could be associated to Bose-Einstein (BE) condensates $[82,83]$ and neutral atoms, which can be used to study entanglement and quantum information processing [84]. Specifically with respect to $\mathrm{BE}$ condensates the idea is to rotate the $\mathrm{BE}$ condensate and to observe the generation of vortices as in $[85,86]$.

Acknowledgments The author would like to thank the referees for useful comments and suggestions. This work was supported in part by means of funds provided by CNPq, Brazil, Grants No. 455719/2014-4 (Universal) and No. 304105/2014-7 (PQ).

Open Access This article is distributed under the terms of the Creative Commons Attribution 4.0 International License (http://creativecomm ons.org/licenses/by/4.0/), which permits unrestricted use, distribution, and reproduction in any medium, provided you give appropriate credit to the original author(s) and the source, provide a link to the Creative Commons license, and indicate if changes were made.

Funded by $\mathrm{SCOAP}^{3}$.

\section{References}

1. G. Petiau, Acad. Roy. de Belg. Classe Sci. Mem in 8o 16, 2 (1936)

2. N. Kemmer, Proc. R. Soc. Lond. A 166, 127 (1938). doi:10.1098/ rspa.1938.0084. http://rspa.royalsocietypublishing.org/content/ 166/924/127.short

3. R.J. Duffin, Phys. Rev. 54, 1114 (1938). doi:10.1103/PhysRev.54. 1114

4. N. Kemmer, Proc. R. Soc. Lond. A 173, 91 (1939). doi:10.1098/ rspa.1939.0131

5. M. Nowakowski, Phys. Lett. A 244, 329 (1998). doi:10.1016/ S0375-9601(98)00365-X. http://www.sciencedirect.com/science/ article/pii/S037596019800365X

6. J.T. Lunardi, B.M. Pimentel, R.G. Teixeira, J.S. Valverde, Phys. Lett. A 268, 165 (2000). doi:10.1016/ 
S0375-9601(00)00163-8. http://www.sciencedirect.com/science/ article/pii/S0375960100001638

7. L.B. Castro, A.S. de Castro, Phys. Rev. A 90, 022101 (2014). doi:10.1103/PhysRevA.90.022101

8. R.F. Guertin, T.L. Wilson, Phys. Rev. D 15, 1518 (1977). doi:10. 1103/PhysRevD.15.1518

9. B. Vijayalakshmi, M. Seetharaman, P.M. Mathews, J. Phys. A: Math. Gen. 12, 665 (1979). doi:10.1088/0305-4470/12/5/015. http://stacks.iop.org/0305-4470/12/i=5/a=015

10. N. Debergh, J. Ndimubandi, D. Strivay, Z. Phys, C 56, 421 (1992). doi:10.1007/BF01565950

11. Y. Nedjadi, R.C. Barrett, J. Phys. A: Math. Gen. 27, 4301 (1994). http://stacks.iop.org/0305-4470/27/i=12/a=033

12. Y. Nedjadi, S. Ait-Tahar, R.C. Barrett, J. Phys. A: Math. Gen. 31, 3867 (1998). http://stacks.iop.org/0305-4470/31/i=16/a=014

13. Y. Nedjadi, R.C. Barrett, J. Phys. A: Math. Gen. 31, 6717 (1998). http://stacks.iop.org/0305-4470/31/i=31/a=016

14. A. Boumali, L. Chetouani, Phys. Lett. A 346, 261 (2005). doi:10. 1016/j.physleta.2005.08.002. http://www.sciencedirect.com/ science/article/pii/S037596010501193X

15. I. Boztosun, M. Karakoc, F. Yasuk, A. Durmus, J. Math. Phys. 47, 062301 (2006). doi:10.1063/1.2203429

16. A. Boumali, Phys. Scr. 76, 669 (2007). http://stacks.iop.org/ $1402-4896 / 76 / \mathrm{i}=6 / \mathrm{a}=014$

17. F. Yasuk, M. Karakoc, I. Boztosun, Phys. Scr. 78, 045010 (2008). http://stacks.iop.org/1402-4896/78/i=4/a=045010

18. A. Boumali, J. Math. Phys. 49, 022302 (2008). doi:10.1063/1. 2841324

19. Y. Kasri, L. Chetouani, Int. J. Theor. Phys. 47, 2249 (2008). doi:10. 1007/s10773-008-9657-6

20. M. Falek, M. Merad, J. Math. Phys. 50, 023508 (2009). doi:10. $1063 / 1.3076900$

21. M. Falek, M. Merad, J. Math. Phys. 51, 033516 (2010). doi:10. $1063 / 1.3326236$

22. M. Falek, M. Merad, Comm. Theor. Phys. 50, 587 (2008). http:// stacks.iop.org/0253-6102/50/i=3/a=10

23. G. Guo, C. Long, Z. Yang, S. Qin, Can. J. Phys. 87, 989 (2009). doi:10.1139/P09-060

24. Z.H. Yang, C.Y. Long, S.J. Qin, Z.W. Long, Int. J. Theor. Phys. 49, 644 (2010). doi:10.1007/s10773-010-0244-2

25. H. Hassanabadi, Z. Molaee, S. Zarrinkamar, Eur. Phys. J. C 72, 2217 (2012). doi:10.1140/epjc/s10052-012-2217-5

26. L.B. Castro, Eur. Phys. J. C 75(6), 287 (2015). doi:10.1140/epjc/ s10052-015-3507-5

27. D.A. Kulikov, R.S. Tutik, A.P. Yaroshenko, Mod. Phys. Lett. A 20, 43 (2005). doi:10.1142/S0217732305016324

28. T.R. Cardoso, L.B. Castro, A.S. de Castro, J. Phys. A: Math. Theor. 43, 055306 (2010). doi:10.1088/1751-8113/43/5/055306. http:// stacks.iop.org $/ 1751-8121 / 43 / \mathrm{i}=5 / \mathrm{a}=055306$

29. T.R. Cardoso, L.B. Castro, A.S. de Castro, Nucl. Phys. B - Proc. Suppl. 199, 203 (2010). Proceedings of the International Workshop Light Cone 2009 (LC2009): Relativistic Hadronic and Particle Physics. doi:10.1016/j.nuclphysbps.2010.02.029. http://www. sciencedirect.com/science/article/pii/S0920563210000496

30. L.B. Castro, A.S. de Castro, Phys. Lett. A 375, 2596 (2011). doi:10.1016/j.physleta.2011.05.067. http://www.sciencedirect. com/science/article/pii/S0375960111006918

31. L.B. Castro, L.P. de Oliveira, AdHEP 2014, Article ID 784072 (2014). doi:10.1155/2014/784072. http://www.hindawi. com/journals/ahep/2014/784072/

32. M. Moshinsky, A. Szczepaniak, J. Phys. A: Math. Gen. 22, L817 (1989). http://stacks.iop.org/0305-4470/22/i=17/a=002

33. P. Strange, Relativistic Quantum Mechanics with Applications in Condensed Matter and Atomic Physics (Cambridge University Press, Cambridge, 1998). doi:10.1017/CBO9780511622755
34. N. Ferkous, A. Bounames, Phys. Lett. A 325, 21 (2004). doi:10. 1016/j.physleta.2004.03.033. http://www.sciencedirect.com/ science/article/pii/S0375960104004128

35. K. Nouicer, J. Phys. A: Math. Gen. 39, 5125 (2006). http://stacks. iop.org $/ 0305-4470 / 39 / \mathrm{i}=18 / \mathrm{a}=025$

36. A.S. de Castro, P. Alberto, R. Lisboa, M. Malheiro, Phys. Rev. C 73, 054309 (2006). doi:10.1103/PhysRevC.73.054309

37. J. Carvalho, C. Furtado, F. Moraes, Phys. Rev. A 84, 032109 (2011). doi:10.1103/PhysRevA.84.032109

38. K. Bakke, C. Furtado, Ann. Phys. (N.Y.) 336, 489 (2013). doi:10. 1016/j.aop.2013.06.007

39. F.M. Andrade, E.O. Silva, M.M.F. Jr., E.C. Rodrigues, Phys. Lett. B 731, 327 (2014). doi:10.1016/j.physletb.2014.02.054. http://www. sciencedirect.com/science/article/pii/S0370269314001531

40. L.B. Castro, A.S. de Castro, P. Alberto, Ann. Phys. (N.Y.) 356, 83 (2015). doi:10.1016/j.aop.2015.02.033. http://www.sciencedirect. com/science/article/pii/S0003491615000925

41. M. Alford, J. March-Russell, F. Wilczek, Nucl. Phys. B 328 , 140 (1989). doi:10.1016/0550-3213(89)90096-5. http://www. sciencedirect.com/science/article/pii/0550321389900965

42. M.G. Alford, F. Wilczek, Phys. Rev. Lett. 62, 1071 (1989). doi:10. 1103/PhysRevLett.62.1071

43. C. Filgueiras, F. Moraes, Phys. Lett. A 361, 13 (2007). doi:10. 1016/j.physleta.2006.09.030. http://www.sciencedirect.com/ science/article/pii/S0375960106014253

44. H. Belich, E.O. Silva, M.M. Ferreira, M.T.D. Orlando, Phys. Rev. D 83, 125025 (2011). doi:10.1103/PhysRevD.83.125025

45. F.M. Andrade, E.O. Silva, M. Pereira, Phys. Rev. D 85, 041701 (2012). doi:10.1103/PhysRevD.85.041701

46. F.M. Andrade, E.O. Silva, M. Pereira, Ann. Phys. (N.Y.) 339, 510 (2013). doi:10.1016/j.aop.2013.10.001. http://www.sciencedirect. com/science/article/pii/S0003491613002157

47. F.M. Andrade, E.O. Silva, Eur. Phys. J. C 74, 3187 (2014). doi:10. 1140/epjc/s10052-014-3187-6

48. G. Sagnac, C. R. Acad. Sci. 157, 708 (1913)

49. G. Sagnac, C. R. Acad. Sci. 157, 1410 (1913)

50. B. Mashhoon, Phys. Rev. Lett. 61, 2639 (1988). doi:10.1103/ PhysRevLett.61.2639

51. L.A. Page, Phys. Rev. Lett. 35, 543 (1975). doi:10.1103/ PhysRevLett.35.543

52. S.A. Werner, J.L. Staudenmann, R. Colella, Phys. Rev. Lett. 42, 1103 (1979). doi:10.1103/PhysRevLett.42.1103. http://link.aps. org/doi/10.1103/PhysRevLett.42.1103

53. J. Anandan, Phys. Rev. D 15, 1448 (1977). doi:10.1103/PhysRevD. 15.1448

54. M. Dresden, C.N. Yang, Phys. Rev. D 20, 1846 (1979). doi:10. 1103/PhysRevD.20.1846

55. J. Anandan, Phys. Lett. A 195(5-6), 284 (1994). doi:10.1016/ 0375-9601(94)90030-2. http://www.sciencedirect.com/science/ article/pii/0375960194900302

56. J.R. Letaw, J.D. Pfautsch, Phys. Rev. D 22, 1345 (1980). doi:10. 1103/PhysRevD.22.1345

57. K. Konno, R. Takahashi, Phys. Rev. D 85, 061502 (2012). doi:10. 1103/PhysRevD.85.061502

58. B.R. Iyer, Phys. Rev. D 26, 1900 (1982). doi:10.1103/PhysRevD. 26.1900

59. G. Vignale, B. Mashhoon, Phys. Lett. A 197(5-6), 444 (1995). doi:10.1016/0375-9601(94)00981-T. http://www.sciencedirect. com/science/article/pii/037596019400981T

60. K. Bakke, Phys. Lett. A 374(46), 4642 (2010). doi:10.1016/ j.physleta.2010.09.046. http://www.sciencedirect.com/science/ article/pii/S0375960110012454

61. K. Bakke, Mod. Phys. Lett. B 27(03), 1350018 (2013). doi:10. 1142/S0217984913500188

62. K. Bakke, Eur. Phys. J. Plus 127(7), 82 (2012). doi:10.1140/epjp/ i2012-12082-2 
63. K. Bakke, Gen. Rel. Grav. 45(10), 1847 (2013). doi:10.1007/ s10714-013-1561-6

64. G. Papini, Phys. Lett. A 377(13), 960 (2013). doi:10.1016/j. physleta.2013.02.032. http://www.sciencedirect.com/science/ article/pii/S0375960113001989

65. U.R. Fischer, N. Schopohl, EPL 54(4), 502 (2001). http://stacks. iop.org/0295-5075/54/i=4/a=502

66. L.H. Lu, Y.Q. Li, Phys. Rev. A 76, 023410 (2007). doi:10.1103/ PhysRevA.76.023410

67. J.Q. Shen, S.L. He, Phys. Rev. B 68, 195421 (2003). doi:10.1103/ PhysRevB.68.195421

68. J.Q. Shen, S. He, F. Zhuang, Eur. Phys. J. D 33(1), 35 (2005). doi:10.1140/epjd/e2005-00027-7

69. J.T. Lunardi, B.M. Pimentel, R.G. Teixeira, Gen. Rel. Grav. 34, 491 (2002). doi:10.1023/A:1015540708007

70. R. Casana, J.T. Lunardi, B.M. Pimentel, R.G. Teixeira, Gen. Rel. Grav. 34, 1941 (2002). doi:10.1023/A:1020732611995

71. E.R.B. de Mello, JHEP 2004(06), 016 (2004). http://stacks.iop.org/ $1126-6708 / 2004 / \mathrm{i}=06 / \mathrm{a}=016$

72. K. Bakke, C. Furtado, Phys. Rev. D 82, 084025 (2010). doi:10. 1103/PhysRevD.82.084025

73. K. Bakke, Int. J. Mod. Phys. A 26(24), 4239 (2011). doi:10.1142/ S0217751X11054437

74. H.F. Mota, K. Bakke, Phys. Rev. D 89, 027702 (2014). doi:10. 1103/PhysRevD.89.027702

75. Y. Nedjadi, R.C. Barrett, J. Phys. G: Nucl. Part. Phys. 19, 87 (1993). doi:10.1088/0954-3899/19/1/006. http://stacks.iop. org $/ 0954-3899 / 19 / \mathrm{i}=1 / \mathrm{a}=006$
76. M. Abramowitz, I.A. Stegun, Handbook of Mathematical Functions (Dover, Toronto, 1965)

77. L.B. Castro, A.S. de Castro, Ann. Phys. (N.Y.) 351, 571 (2014). doi:10.1016/j.aop.2014.09.012. http://www.sciencedirect. com/science/article/pii/S0003491614002723

78. D.R. Nelson, Defects and Geometry in Condensed Matter Physics (Cambridge University Press, Cambridge, 2002)

79. T. Senthil, M. Levin, Phys. Rev. Lett. 110, 046801 (2013). doi:10. 1103/PhysRevLett.110.046801

80. A. Sterdyniak, N.R. Cooper, N. Regnault, Phys. Rev. Lett. 115, 116802 (2015). doi:10.1103/PhysRevLett.115.116802

81. G. Palumbo, K. Meichanetzidis, Phys. Rev. B 92, 235106 (2015). doi:10.1103/PhysRevB.92.235106

82. R. Casana, V.Y. Fainberg, B.M. Pimentel, J.S. Valverde, Phys. Lett. A 316(1), 33 (2003). doi:10.1016/S0375-9601(03)01018-1. http:// www.sciencedirect.com/science/article/pii/S0375960103010181

83. L.M. Abreu, A.L. Gadelha, B.M. Pimentel, E.S. Santos, Phys. A: Stat. Mech. Appl. 419(0), 612 (2015). doi:10.1016/j.physa. 2014.10.049. http://www.sciencedirect.com/science/article/pii/ S0378437114008875

84. D. Jaksch, H.J. Briegel, J.I. Cirac, C.W. Gardiner, P. Zoller, Phys. Rev. Lett. 82, 1975 (1999). doi:10.1103/PhysRevLett.82.1975

85. G. Baym, C.J. Pethick, Phys. Rev. Lett. 76, 6 (1996). doi:10.1103/ PhysRevLett.76.6

86. E. Lundh, C.J. Pethick, H. Smith, Phys. Rev. A 55, 2126 (1997). doi:10.1103/PhysRevA.55.2126 